\title{
Achieving of intensified conductive interconnections for Flex-on-Flex by using metal passivated Copper - Copper Thermocompression bonding
}

\author{
C. Hemanth Kumar,* Asisa Kumar Panigrahi, \\ Satish Bonam, Nirupam Paul, Siva Rama Krishna \\ Vanjari, and Shiv Govind Singh \\ Department of Electrical Engineering \\ Indian Institute of Technology Hyderabad \\ Sangareddy, India \\ *ee16resch01006@iith.ac.in
}

\author{
Asisa Kumar Panigrahi \\ Department of Electronics \& Communication \\ Engineering, \\ KL University Hyderabad, Hyderabad, India \\ asisa@kluniversityh.in
}

\begin{abstract}
There is a gradual increase in demand for flexible electronics due to the way it is going to empower the end user to bent, roll/fold and arrange randomly in 3-D space, the devices without sacrificing the performance and reliability of devices, in a trend focused towards ever shrinking device footprint area. One of the prime mover towards realizing flexible electronics is interconnect scaling, which is motivating us to move towards three-dimensional interconnect integration. In this paper, we propose the interconnection of two flexible-flexible substrates by Thermocompression bonding between copper-to-copper, with their surface passivated with palladium, at low temperature and low pressure, to overcome the limitations imposed by conventional anisotropic conductive film (ACF) and nonconductive paste (NCP) approaches. The enhancement in interdiffusion of atomic species between the two surfaces, at low temperature and pressure, is possible only with unoxidized surface which has low RMS roughness or with surface which has varied film density. We have systematically optimized the thickness of palladium passivation layer for high quality $\mathrm{Cu}-\mathrm{Cu}$ bonding, at low temperature of $140{ }^{\circ} \mathrm{C}$ and at low pressure of 5 bar. 2.06 nm RMS surface roughness was observed for Palladium passivation layer when its thickness was optimized at $5 \mathrm{~nm}$, which made bonded $\mathrm{Cu}-\mathrm{Cu}$ junction interface free from any copper oxide, as it was confirmed by X-ray Diffraction (XRD) and Energy Dispersive Spectroscopy (EDS) analysis. To confirm the quality of bonded interface, the samples were subjected to stress by folding just after Thermocompression bonding. Absence of any voids between the interfaces during Scanning Acoustic Microscopy (SAM) imaging confirms the superior quality of $\mathrm{Cu}-\mathrm{Cu}$ bonding. The bonded interface image from cross-sectional Scanning Electron Microcopy (XSEM) further reaffirms the quality of interface. Besides, very low contact resistance of $\sim 2$ E-7 $\Omega-\mathrm{cm}^{2}$ obtained for a fabricated daisy chain pattern with $70 \mathrm{um}$ pitch and $100 \mathrm{um} \times$ $100 \mathrm{um}$ bonding contact, in addition, confirms the good quality of bonding interface. The demonstrated bonding approach with metal passivated interconnect technique will be one of the prime contestant for future high bandwidth applications.
\end{abstract}

Keywords-Thermocompression bonding, surface passivation, RMS surface rouhness, atomic inter diffusion.

\section{INTRODUCTION}

There will a greater need of flexible and bendable electronics in the future, for wearable and Internet-of-Things
(IoT) applications, for RFIDs, sensors, displays and memory devices, to interconnect every object in the surrounding in a single network in a most cost effective manner [1].

These fascinating services would be possible only with very high circuit functionality, when they are being integrated on flexible substrate by incorporating diverse electronic circuits with devices. Traditionally, due to the absence of advanced technology to make direct electronic devices on flexible substrate, it has evolved from heterogeneous package integration of dice with flexible substrate, called Chip-on-Flex (COF) or die embedded in flex, known as Chip-in-Flex (CIF), by using either anisotropic conductive film (ACF) ${ }^{[2]}$ or non-conductive paste (NCP) approaches. In conventional ACF technique, polymer resins with conductive particles are used in between the interconnections, which provides the electrical paths in the respective package ${ }^{[3]}$. Whereas in NCP approach, a nonconductive epoxy resins without conductive particles are used for die interconnections on flexible substrate, for high mechanical strength and stable contact resistance, besides providing high peel strength over the greater temperature range. The main limitations of these conventional approaches are the need for critical curing processes, or else, there will be a severe reliability issues, and the difficulty to scale-down interconnect pitches, which acts as a major bottleneck towards attaining a high density interconnects between substrates.

As ACF uses resins with conductive particles, thus special attention has to be given in selecting conductive particle density. If the particle density is higher, the interlayer short circuits the interconnects, or else interlayer resistance will be higher. One more crucial factor for higher interconnect density with ACF is size of conductive particles. In the NCP approach, there is a need of making the large contact area for higher reliability. Because of all these limitations in the conventional approaches, flexible interconnection packaging seems to be the most viable alternative. Flex-On-Flex (FOF) interconnection approach is one of the most cost-effective alternative towards realizing the next generation interconnect technology. FOF interconnection by three-dimensional integration will be an excellent choice for achieving high interconnect density with flexible hybrid assemblies [4].

To make these interconnections between the substrates, the conventional mechanism is metallic bonding, whereas in 
case of ACF and NCP approaches, the mounting of dies or substrates having metallic bump in accordance with another substrate with a flexible interlayer resin is needed, the limitations of which has already been discussed earlier.

One of the appreciable solution for this is direct metallic bonding at low temperature. K.N. Chen and his group has developed eutectic based flex-on-flex bonding [5], in which there is a possibility of formation of intermetallic compound at the junction interface. These intermetallic compounds are very well known for their brittle nature, which may degrade the conductivity of inter-connections. Therefore, there is an overwhelming necessity to look for some suitable alternatives that will provide excellent interconnect with very high interconnect conductivity and with greater reliability, realizing which is a great challenge in itself.

Best economical solution towards this will be, to use copper for direct metallic interconnections ${ }^{[6]}$. There is a need for effective interdiffusion of copper atoms to make higher grains. Proper interdiffusion of copper atoms at the surface is a major challenge, which is limited by two factor, first is the formation of oxide compounds on the copper surface during bonding and second is the requirement for nanometer of surface roughness at the top surface ${ }^{[8]}$.

In this work, we proposed an approach, by which it is possible to make direct metal-to-metal $(\mathrm{Cu}-\mathrm{Cu})$ bonding interconnections by inter-diffusion of metallic atom in between the flexible substrates using another metal as a passivation layer, thickness of which is optimized for achieving the low temperature and low pressure Thermocompression bonding.

\section{ThE BONDING MECHANISM AND BONDING PROCESS}

\section{A. Optimization of Passivation layer thickenss of Pd layer}

In the case of thermocompression bonding, as mentioned above, one of the major bottle neck is surface roughness. If the surface roughness is more, higher bonding pressure will be required due to uneven troughs/crests on the $\mathrm{Cu}$ surface. Thus, passivation material have dual role to play, firstly, it has to protect the $\mathrm{Cu}$ surface from getting oxidized as informed previously apart from providing ${ }^{[9]}$ smooth surface with very minimal RMS roughness. Hence, thickness of palladium will play a key role in achieving good surface passivation in protecting copper surface from getting oxidized towards achieving better $\mathrm{Cu}-\mathrm{Cu}$ bonding interface and lower interface contact resistance. Since the resistivity of $\mathrm{Pd}$ is one order higher than $\mathrm{Cu}$, therefore, thicker Pd layer, may affect the contact resistance at the bonding interface. Hence, there is a need for optimizing the thickness of palladium passivation layer for high quality and reliable $\mathrm{Cu}-\mathrm{Cu}$ bonding interface, for multilayer integration.

To evaluate surface roughness of the passivated $\mathrm{Cu}$ film, we deposited Pd passivation film multiple times, each time varying the $\mathrm{Pd}$ thickness from $1 \mathrm{~nm}$ to $7 \mathrm{~nm}$, over $\mathrm{Cu}$ surface $(200 \mathrm{~nm})$. The surface roughness of the prepared samples were then determined using Atomic Force
Microscope (AFM) under tapping mode with sample scan are of $5 \mathrm{um}$.

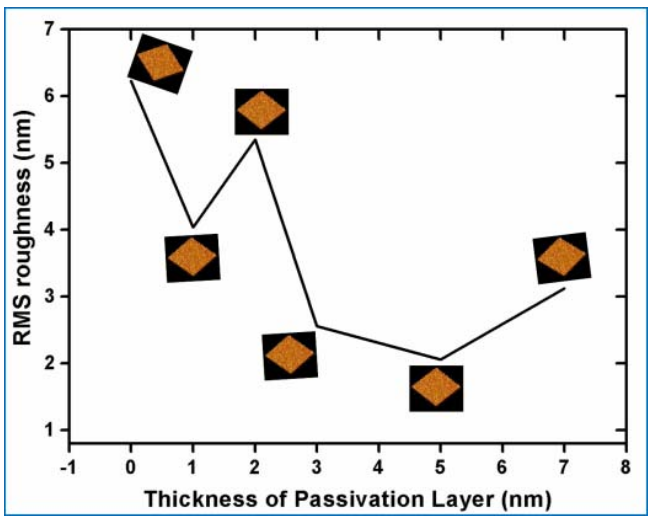

Figure 1. Deviation of RMS roughness on $\mathrm{Cu}$ surface with varying Palladium (Pd) passivation layer.

Figure 1, shows variation of RMS roughness of $\mathrm{Pd}$ passivated $\mathrm{Cu}$ substrates with varying $\mathrm{Pd}$ film thicknesses. Passivated $\mathrm{Cu}$ surface with $5 \mathrm{~nm}$ Pd ultrathin film has the lowest RMS roughness of $2.06 \mathrm{~nm}$, which is comparatively much lower than other passivated $\mathrm{Cu}$ samples.

After finding out minimal surface roughness with $5 \mathrm{~nm}$ passivation layer on $\mathrm{Cu}$ surface, the effectiveness of optimized layer to be tested in such a way that can it will be able to compensate the surface oxide prevention or not, to observe this issue, has been explained in preponing analysis of copper surface.

\section{B. Bonding Mechanism and Pre Bonding analysis of copper surface}

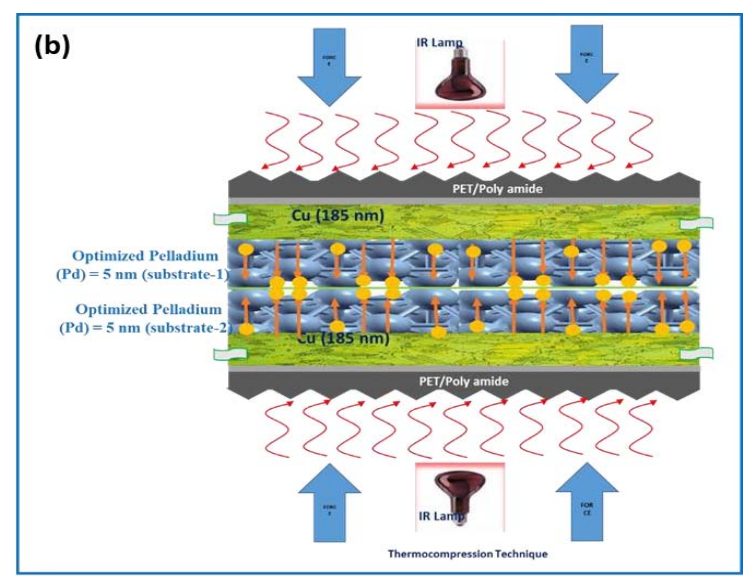

Figure 2. The bonding process in passivated themocomperssion cycle.

Palladium has been used in this method as an optimized thick passivation material due to its high temperature oxidation resistive properties ${ }^{[10]}$, relatively good electrical conductivity at room temperature. Apart from these, palladium has comparably low density than $\mathrm{Cu}$ and it has 
higher atomic radius of $1.37 \mathrm{~A}^{\circ}$ respectively than atomic radius of pure $\mathrm{Cu}\left(1.28 \mathrm{~A}^{\circ}\right)$. This is one of the key aspect that tells us about the surface compressive stress by the higher atomic radius atoms, on the copper surface makes easier for inter diffusion. In addition, $\mathrm{Cu}$ is having lower activation energy at the surface [11]. Hence, $\mathrm{Cu}$ can easily diffuse through palladium by applying of temperature and pressure while thermocompression bonding and interdiffusion and surface stresses with themocomperssion boding process as shown in Figure 2. From this due to surface stresses and having the path for the interdiffusion of copper atoms, by applying the inadequate pressure and temperature makes the surface atoms causes the moving towards each other and make the sophisticated grains at the interface caused the high strength interconnects with the high reliable and effective conductive paths.

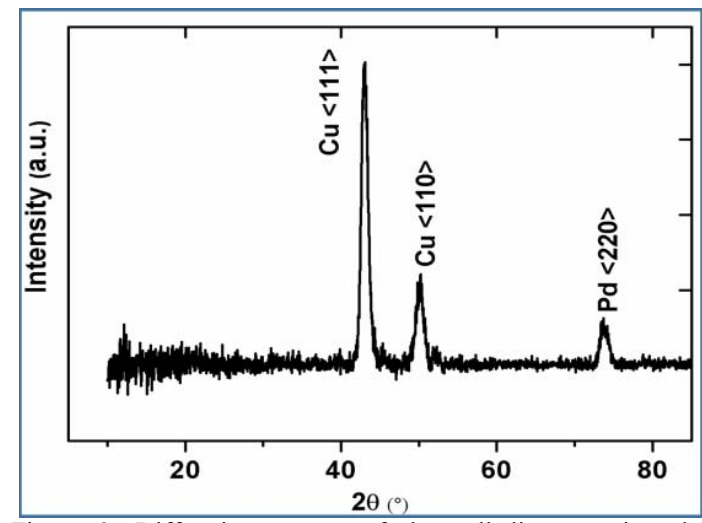

Figure 3. Diffraction pattern of the palladium passivated $\mathrm{Cu}$ surface Reflection of pure $\mathrm{Cu}<111>$ orientation plane is an indicative of diffusion enhancement.

Further, surface diffusion coefficient at the bonding interface plays vital role in enhancement of interdiffusion. Surface diffusion coefficient of $<111>$ oriented $\mathrm{Cu}$ plane is 3 to 4 orders of higher magnitude compared to other oriented $\mathrm{Cu}$ plane at the bonding interface ${ }^{[12]}$. In order to evaluate the preferable $\mathrm{Cu}$ plane orientation at the surface of an optimized layer of passivated $\mathrm{Cu}$ surface was subjected to same thermal stress as that of bonding conditions and subsequent Glancing angle X-Ray Diffraction (GI-XRD) was performed. Figure 3, confirms the reflections of pure $\mathrm{Cu}<111>$ plane along with frail $\mathrm{Cu}<110>$ and $\mathrm{Pd}<220>$.

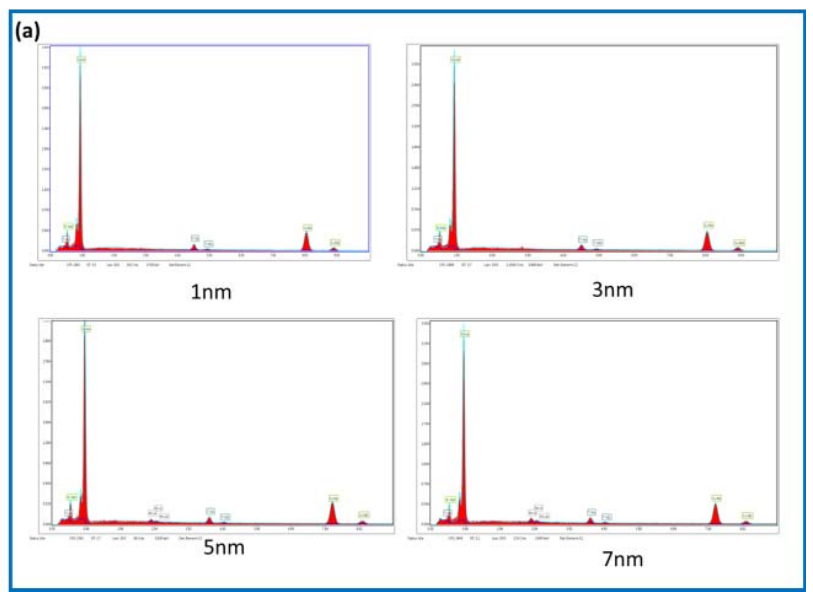

From the observation of XRD, we got the conformation that for an optimized thickness of Pd layer of $5 \mathrm{~nm}$ acted as a surface oxide barrier for copper. This enhanced diffusion caused by surface stress and high surface diffusion coefficient enables low temperature and low pressure high quality bonding.

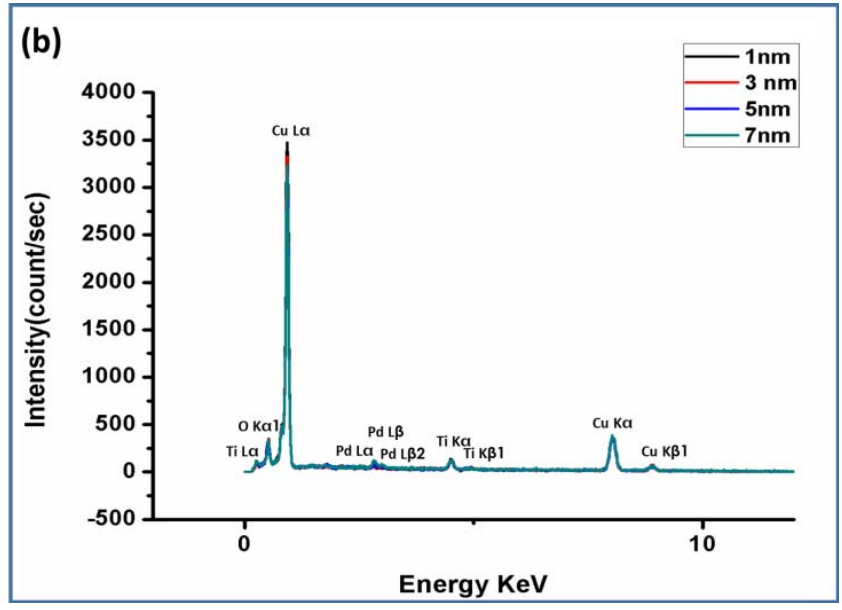

Figure 4. (a) EDS analysis of passivated samples (b) EDS comparison plot

To conform the surface oxide on the copper surface we have been performed the energy dispersive X-Ray spectroscopy (EDS) by using the scanning electron microscopy (SEM) for the samples with ultrathin $\mathrm{Pd}$ passivation layer of 1, 3, 5 and $7 \mathrm{~nm}$ as shown in Figure 4(a). We observed the reduced oxygen on the sample on the $5 \mathrm{~nm}$ ultrathin $\mathrm{Pd}$ passivated copper film, we observed the common oxide peak on each sample because of chamber ambient oxide in instrument. The comparison plot of EDS profile of the samples are shown in the Figure 4(b).

\section{Experimental}

Here we have taken the clean $5.5 \mathrm{~cm} \times 5.5 \mathrm{~cm}$ square samples of flexible substrates (PET/ Kapton Polyamide) for the face to face $\mathrm{Cu} / \mathrm{Pd}-\mathrm{Pd} / \mathrm{Cu}$ blanket bonding. Initially flex films were cleaned with acetone, via ultrasonicator for 5 mins, followed by isopropanol alcohol (IPA), followed by bake, up to 2 mins at $100^{\circ} \mathrm{C}$ in order to retain the plane surface and complete removal of wet content present on the substrates. Then on the cleaned flex surfaces, thin film deposition was carried by $20 \mathrm{~nm}$ of $\mathrm{Ti}$ as an adhesive layer for copper on flex, $200 \mathrm{~nm}$ of $\mathrm{Cu}$ layer deposition followed by optimized palladium passivation layer of $5 \mathrm{~nm}$. Then the samples carried for the bonding. 


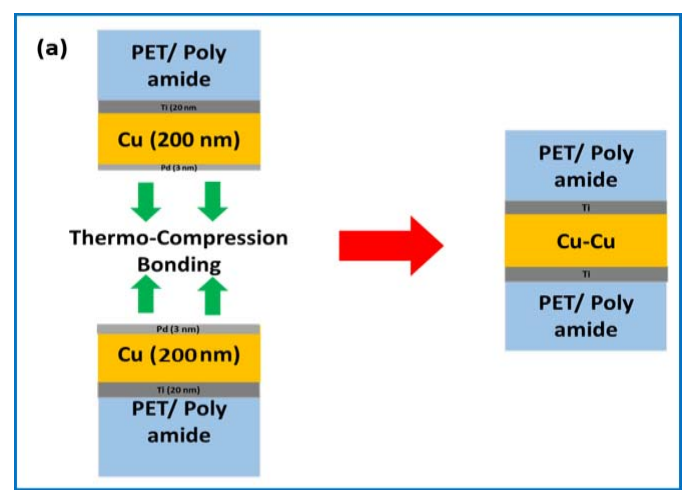

Figure 5. The bonding mechanism

Bonding was performed inside the alignment Wafer Bonder (AML-AWB, UK). The thin film deposited flexible substrates kept face-to-face and closed the chamber, before the creation of vacuum inside the bonding chamber. Once the vacuum created up to $5 \times 10^{-5}$ torr, then the heater was turned on. Once the temperature reached to $140^{\circ} \mathrm{C}$, we applied a contact force of $4 \mathrm{kN}$ was applied for $50 \mathrm{mins}$. Then the bonded flexible films are kept steady inside the bonding chamber in order to cool down to room temperature. Figure 5 , briefly describes the schematic of bonding process using thermocompression cycle. Then various post bonding characterizations were performed in order to evaluate the bonding quality which discussed briefly in the further results and discussion section.

\section{RESULTS AND DISCUSSION}

\section{A. Bondigng ineface by bending angle analysis.}

The bonded flexible substrates, can be expected for the bending angles, to test the quality of bond we have tried manually to bend up to $\sim 45^{\circ}$ bending of bonded samples. We observed the good bonding quality and we used razor blade test at the inter face to peel-off the bonded samples, but we observed the good bonding at the interface.
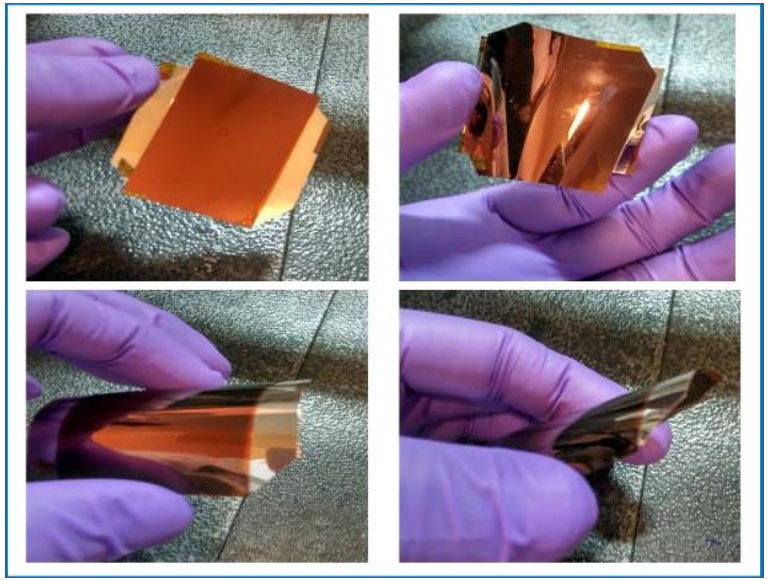

Figure 6 . Testing of bonding quality of flexible substrates by bending.

\section{B. Bondign interface with Scanning acoustic microscopy observation}

The copper bonded samples on flexible substrates with 5 $\mathrm{nm}$ passivated samples are subjected to scanning acoustic microscopy (SAM) as shown in the Figure 7. To perform this, we have taken $5.5 \mathrm{~cm} \times 5.5 \mathrm{~cm}$ bonded samples in Figure 7(a), has attached to stage in Figure 7(b) with surrounded tape to protect the damaging of sample under water and we kept it in water tub having acoustic transceiver shown in Figure 7(c) from D9600 C-SAM, and we went for imaging using appropriate peak. We observed the interface is a void free and high efficient bonding quality as shown in Figure 7(d)

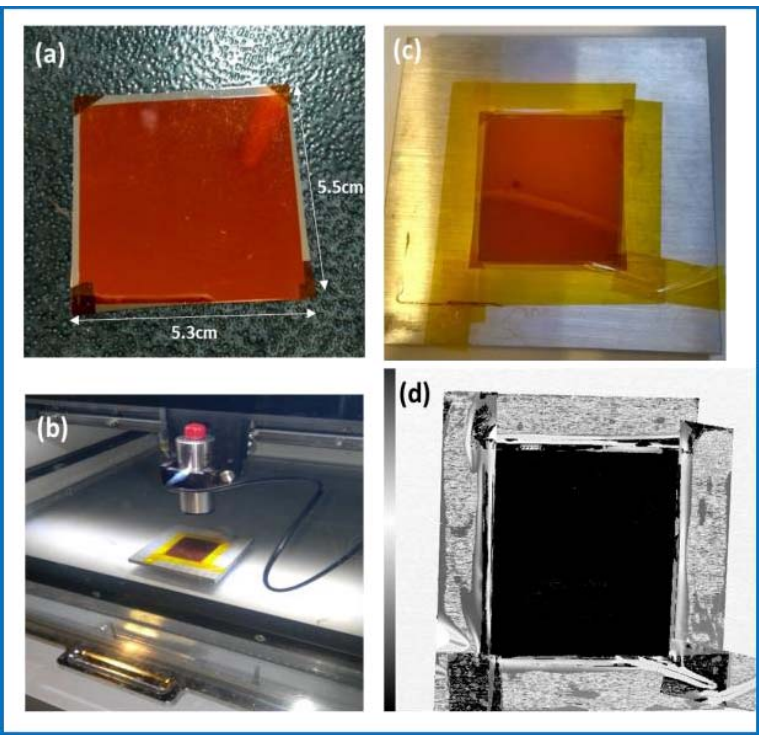

Figure 7. SAM analysis of bonded samples

\section{Microstructure imaging of the bonded substrates}

Cross-sectional imaging analysis basically useful for the assessment of bonded intermediate interface layer excellence. Undesirable intermediate layer or uneven voids at the interface reduce the bonding quality. In order to identify the presence of intermediate layers and voids, Field Emission-Single Electron Microscopy (FE-SEM, ZEISS) was carried out. Figure 8, shows the cross section FE-SEM image of the bonded layer. The picture as shown is confirms the success of $\mathrm{Cu}-\mathrm{Cu}$ bonding with optimized palladium passivation. The presence of fine bonding between two $\mathrm{Cu}$ films with palladium passivation is a proof for high quality bonding. 


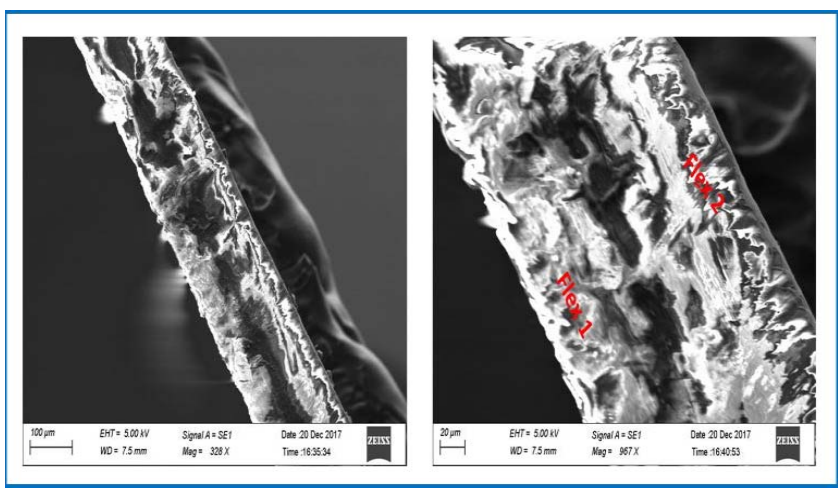

Figure 8. FE-SEM analysis of bonded interface.

\section{Electrical analysis.}

The electrical characterization has been performed using modified kelvin structure as shown in Figure 9.

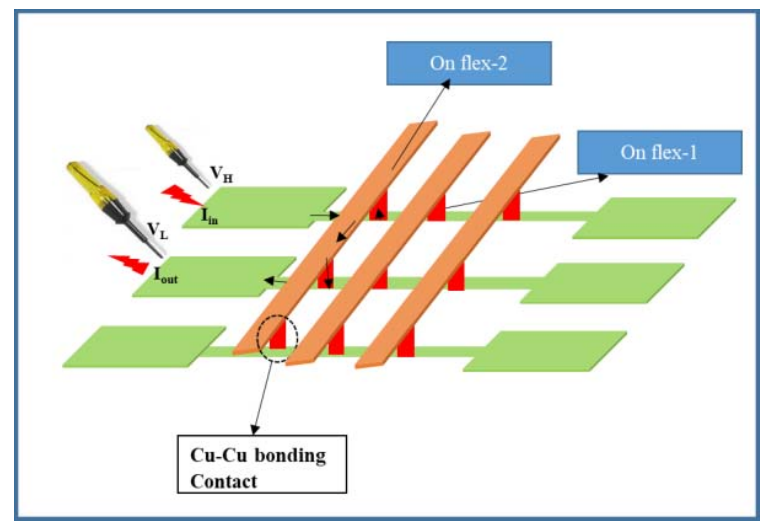

Figure 9. Modified kelvin structure.

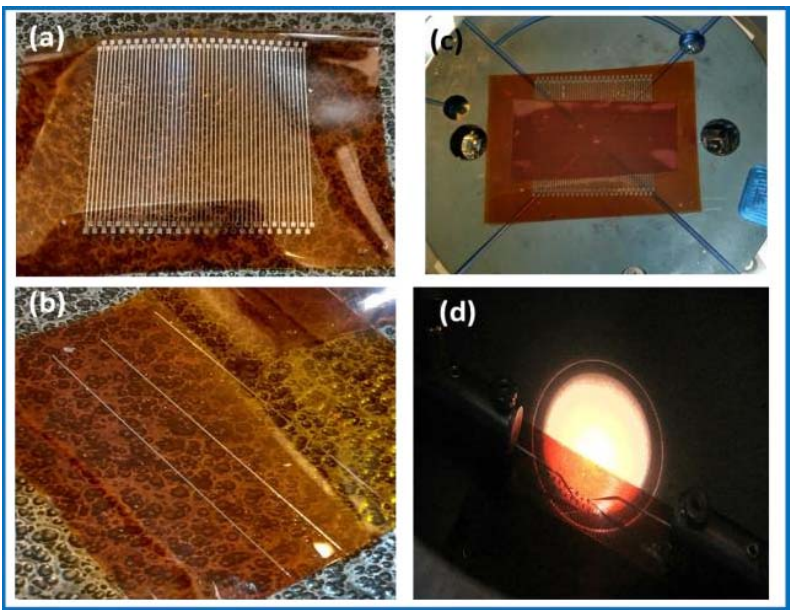

Figure 10. (a) Contact lines with pads on flex 1 (b) contact lines on flex 2 (c) Bonded sample (d) bonded sample under test.
Here we have fabricated $70 \mathrm{um}$ pitch of contact lines with bond pads on one flexible substrate with 100 um width shown in Figure 10(a), and 100 um width of metallic lines were fabricated as shown in Figure 10(b) on one more flexible substrate by using the lithographic process, after ultrasonic cleaning in acetone followed by drying and followed by spin coating of S1813 photoresist, followed by writing with laser beam with laser power of $812 \mathrm{~mJ} / \mathrm{cm}^{2}$.

After writing the patterns we kept the sample in acetone for 5 hours, for lift off. Then we have done utlrasonication for $10 \mathrm{sec}$. we find the good patterns on flexible substrate. After sample preparation, we went for bonding we have done it for $50 \mathrm{~min}$ at $3.5 \mathrm{kN}$ and take out the sample for electrical characterization using Keithley 4200A-SCS Parameter Analyzer, and we applied the voltage and respective current readings the specific contact resistivity has been found at plane condition and $30^{\circ}$ bending is $\sim 2 \mathrm{E}-7$ $\Omega-\mathrm{cm}^{2}$. This high conductive inter connects are novel mothed for motivating future flex on flex interconnections.

\section{CONCLUSION}

Flex-on-Flex $\mathrm{Cu}-\mathrm{Cu}$ thermocompression bonding was successfully demonstrated at $140^{\circ} \mathrm{C}$ with nominal contact force of $4 \mathrm{kN}$, using optimally chosen ultra-thin Palladium passivation layer of $5 \mathrm{~nm}$ thickness. The proposed bonding technology is suitable for Flex-on-Chip (FOC) and Flex-inChip (FIP) applications as-well-as for high bandwidth applications like IoT built on Flex-on-Flex technology in a cost effective manner. Furthermore, SAM imaging of Flexon-Flex bonded sample just after bending test, shows a void free interface, which confirms good quality interface, as also confirmed by X-SEM analysis. In addition, modified Kelvin daisy-chain analysis of passivated bonded structure provides specific contact resistance of $\sim 2.0 \mathrm{E}-7 \Omega-\mathrm{cm}^{2}$, for normal and bending conditions. This suggests excellent contact stability without any electrical performance degradation. It is hoped that the presented result will pave the way for future flexible high conductive interconnections.

\section{ACKNOWLEDGMENT}

Authors are grateful for the financial support from the Department of electronics and information technology (Deity), Government of India.

\section{REFERENCES}

[1] Zhan, Yiqiang, Yongfeng Mei, and Lirong Zheng. "Materials capability and device performance in flexible electronics for the Internet of Things." Journal of Materials Chemistry C 2.7 (2014): 1220-1232.

[2] Suk, K. L., Son, H. Y., Chung, C. K., Do Kim, J., Lee, J. W., \& Paik, K. W. (2012). Flexible Chip-on-Flex (COF) and embedded Chip-inFlex (CIF) packages by applying wafer level package (WLP) 
technology using anisotropic conductive films (ACFs). Microelectronics Reliability, 52(1), 225-234.

[3] ] Liang, R. C., Chung, J., Tseng, C., Rokutanda, S., Sun, Y., \& Chuang, H. K. (2014). U.S. Patent No. 8,802,214. Washington, DC: U.S. Patent and Trademark Office.

[4] Meyer, J. U., Stieglitz, T., Scholz, O., Haberer, W., \& Beutel, H. (2001). High density interconnects and flexible hybrid assemblies for active biomedical implants. IEEE Transactions on Advanced Packaging, 24(3), 366-374.

[5] Hu, Y. C., \& Chen, K. N. (2016). A Novel Bonding Approach and Its Electrical Performance for Flexible Substrate Integration. IEEE Journal of the Electron Devices Society, 4(4), 185-188.

[6] Gupta, Tapan. Copper interconnect technology. Springer Science \& Business Media, 2010.

[7] Kim, T. H., Howlader, M. M. R., Itoh, T., \& Suga, T. (2003). Room temperature $\mathrm{Cu}-\mathrm{Cu}$ direct bonding using surface activated bonding method. Journal of Vacuum Science \& Technology A: Vacuum, Surfaces, and Films, 21(2), 449-453.
[8] Gueguen, P., di Cioccio, L., Rivoire, M., Scevola, D., Zussy, M., Charvet, A. M., ... \& Clavelier, L. (2008, June). Copper direct bonding for 3D integration. In Interconnect Technology Conference, 2008. IITC 2008. International (pp. 61-63). IEEE.

[9] Optimized ultra-thin manganin alloy passivated fine-pitch damascene compatible bump-less $\mathrm{Cu}-\mathrm{Cu}$ bonding at sub $200{ }^{\circ} \mathrm{C}$ for threedimensional Integration applications, February 2018, Japanese Journal of Applied Physics 57(2S1):02BC04

[10] Greenwood, Norman N. Earnshaw, Alan, "Chemistry of the Elements", Oxford, Pergamon Press, pp. 1336-37, (1984).

[11] Agrawal PM, Rice BM, Thompson DL. Predicting trends in rate parameters for self-diffusion on FCC metal surfaces. Surface Science. 2002 Aug 1;515(1):21-35.

[12] Liu, Chien-Min, Han-wen Lin, Yi-Cheng Chu, Chih Chen, DianRong Lyu, Kuan-Neng Chen, and K. N. Tu. "Low-temperature direct copper-to-copper bonding enabled by creep on highly $\left(\begin{array}{lll}1 & 1 & 1\end{array}\right)$-oriented Cu surfaces." Scripta Materialia 78 (2014): 65-68. 